\title{
Overexpression of the prolyl isomerase PIN1 promotes cell growth in osteosarcoma cells
}

\author{
LU ZHOU $^{1 *}$, BYUNG-HYUN PARK $^{2 *}$, JONG HYUK PARK $^{1}$, KYU YUN JANG $^{3}$, HO SUNG PARK $^{3}$, \\ SAJEEV WAGLE ${ }^{1}$, KWANG-BOK LEE ${ }^{1}$ and JUNG RYUL KIM ${ }^{1,4}$
}

\begin{abstract}
Departments of ${ }^{1}$ Orthopaedic Surgery, ${ }^{2}$ Biochemistry and ${ }^{3}$ Pathology, Chonbuk National University Medical School; ${ }^{4}$ Research Institute for Endocrine Sciences, Chonbuk National University, Jeonju, Jeonbuk 561-756, Republic of Korea
\end{abstract}

Received September 3, 2012; Accepted October 19, 2012

DOI: $10.3892 /$ or.2012.2112

\begin{abstract}
PIN1 was recently identified as a peptidyl-prolyl cis-trans isomerase (PPIase). It binds to and isomerizes specific pSer/Thr-Pro motifs and catalytically induces conformational changes after phosphorylation. PIN1 plays an important role in several cellular events, such as cell cycle progression, transcriptional regulation, RNA processing, cell proliferation and differentiation. The relationship between PIN1 and osteosarcoma has not been previously studied. In the present study, we investigated the expression pattern of PIN1 in human osteosarcoma tissues and the role of PIN1 in osteosarcoma generation and development. The expression levels of PIN1 were detected by immunohistochemistry and western blotting. Results demonstrated that the expression of PIN1, cyclin D1 and $\beta$-catenin were significantly higher in human osteosarcoma tissues compared to normal tissues. The in vitro effects of PIN1 overexpression were studied in human osteosarcoma cell lines. Adenovirus-mediated PIN1 overexpression significantly stimulated the proliferation of MG-63 and U2-OS osteosarcoma cells by $148 \pm 10.5$ and $187 \pm 21.5 \%$, respectively. In FACS analysis, U2-OS cells displayed significant levels of arrest in cell cycle progression at the $G_{0} / G_{1}$ phase. Consistent with increased cell growth, levels of cyclin D1 and cyclin E and their associated cyclin-dependent kinases, CDK4 and CDK6, were enhanced in PIN1-overexpressed cells compared with the control virustransfected cells. When the PIN1 inhibitor juglone was added to the cells, the proliferative effects of PIN1 were abolished. These results suggest that PIN1 may play an important role in tumorigenesis and tumor progression of osteosarcoma and therefore, provide a new target for gene therapy.
\end{abstract}

Correspondence to: Professor Jung Ryul Kim, Department of Orthopaedic Surgery, Chonbuk National University Medical School, 567 Baekje-daero, Deokjin-gu, Jeonju, Jeonbuk 561-756, Republic of Korea

E-mail: jrkeem@jbnu.ac.kr

*Contributed equally

Key words: PIN1, osteosarcoma, proliferation, cell cycle, cyclin D1

\section{Introduction}

Osteosarcoma is the most common primary bone tumor that occurs mainly in children and adolescents. Twenty percent of osteosarcoma patients succumb to this disease as a result of tumor metastasis or an unresectable tumor. The remaining $80 \%$ of patients generally present with small metastases when diagnosed; a number of these patients develop pulmonary metastasis within a year and the 5-year survival rate is only $15 \%(1,2)$. Recently, various chemotherapies and new diagnostic techniques have been developed. These developments have dramatically improved the 5-year survival rate for osteosarcoma patients up to $55-70 \%$ (1). Chemotherapeutic regimens used in the treatment of osteosarcoma, such as adriamycin, methotrexate and/or cisplatin, result in significant morbidity, including cardiac toxicity, infertility and renal dysfunction. In order to improve the long-term survival rate of this type of cancer, it is crucial for research efforts to identify new targets and techniques, especially for gene therapy.

It has been suggested that protein phosphorylation is a critical step in protein structural change. PIN1 is composed of 2 functional domains, an amino-terminal WW domain (amino acids 1-39) involved in protein-protein interaction which mediates PIN1 binding to phosphorylated protein and a COOH-terminal peptidyl-prolyl cis-trans isomerase (PPIase) domain (amino acids 45-163) that functions in catalysis (3). Phosphorylation of Ser/Thr-Pro residues plays an important role in several cellular events. An anomalous change in the protein during the process of signal transduction usually leads to unwanted cell proliferation and culminates in the development of cancer $(4,5)$.

PIN1 was originally identified by a yeast 2-hybrid screen as a protein that interacts with NIMA (never in mitosis gene A). It consists of 163 amino acid residues and is located at $19 \mathrm{p} 13(6,7)$. It has been reported that PIN1 is involved in several aspects of signal transduction, such as cell growth, proliferation, differentiation and apoptosis and is upregulated in various types of tumor tissues. Currently, approximately 20 types of PIN1 targets are known, such as p53, $\beta$-catenin and cyclin D1, and most are related to the development of tumors $(8,9)$. PIN1 has important effects in a number of carcinogenic signaling pathways. It catalyzes the composite of a carcinogenic substance and prevents the degradation of the carcinogenic substance. 
Thus, PIN1 as an amplifier of carcinogenic signals, plays a crucial role in the process of transforming an oncogene to a signal of cell proliferation and differentiation.

Cyclin D1 is one of the important targets of PIN1. PIN1 affects not only the Ras/JNK pathway to accelerate the synthesis of cyclin D1 during transcription (10), but also prevents the degradation of cyclin D1 by changing the structure of cyclin D1 to block its movement from the nucleolus to the cytoplasm (11). $\beta$-catenin is a crucial downstream transcriptional factor of the Wnt signaling pathway, and also improves cyclin D1 synthesis. PIN1 specifically binds to the phosphorylated motif of $\beta$-catenin, altering its configuration and thus inhibiting the combination of $\beta$-catenin and APC. PIN1 also improves the expression of cyclin D1 by increasing the stability of $\beta$-catenin and its accumulation in the nucleolus (9). Cyclin D1 is a member of the cyclin family, and is known to catalyze the $\mathrm{G}_{1}-\mathrm{S}$ phase transition. Cyclin D1 phosphorylates Rb protein by activating CDK4 and CDK6. The phosphorylated Rb protein releases E2F, which initiates DNA transcription and induces the transition from the $G_{1}$ to $S$ phase. The overexpression of cyclin D1 leads mammary glandular cells to transform into inocytes $(12,13)$ and promotes breast cancer formation (14). Together, these reports strongly suggest that PIN1 and cyclin D1 play an important role in the occurrence and development of tumors.

The expression of PIN1 in human breast and oral cancers is closely correlated with the cyclin D1 level $(15,16)$. However, the relationship between PIN1 and osteosarcoma has not been previously studied. Therefore, we studied the expression patterns of PIN1 in human osteosarcoma tissues. We further investigated the effect of the overexpression of PIN1 in osteosarcoma cells on cell proliferation and cycle. Our data demonstrated that PIN1 traverses the $\mathrm{G}_{0}-\mathrm{G}_{1}$ and/or $\mathrm{G}_{1}-\mathrm{S}$ transitions through a coordinated mechanism involving the upregulation of cyclins and CDKs and the downregulation of $\mathrm{p} 21$.

\section{Materials and methods}

Cell culture and reagents. Human osteosarcoma MG-63 and U2-OS cells were purchased from the American Type Culture Collection (ATCC, Rockville, MD, USA) and HEK293T cells were maintained in our laboratory. The cells were grown at $37^{\circ} \mathrm{C}$ in a humidified atmosphere of $5 \% \mathrm{CO}_{2}$ in DMEM medium supplemented with $10 \%$ fetal bovine serum and $2 \mathrm{mM}$ glutamine, $100 \mathrm{U} / \mathrm{ml}$ penicillin, $100 \mu \mathrm{g} / \mathrm{ml}$ streptomycin and $2.5 \mu \mathrm{g} / \mathrm{ml}$ amphotericin B. All reagents were purchased from Sigma (St. Louis, MO, USA) unless otherwise noted.

Tissue preparation. Eight paraffin samples of human osteosarcoma and normal tissues were obtained from Chonbuk National University Hospital after Ethics Committee approval of the use of human tissue. Tissues were fixed in a $4 \%$ formalin solution and embedded in wax blocks. Each block was cut into $5-\mu \mathrm{m}$ sections and stained with specific antibodies. Eight cases of fresh human osteosarcoma and normal tissues $\left(5-8 \mathrm{~mm}^{3}\right)$ were acquired during surgery and stored at $-80^{\circ} \mathrm{C}$ until use.

Transfection of cells. Preparation and amplification of the adenovirus were performed as previously described (17). Adenoviruses encoding PIN1 were created using the Virapower Adenoviral Expression System according to the manufacturer's instructions (Invitrogen, Carlsbad, CA, USA). Site-specific recombination between entry vectors (PIN1-pENTR) and the adenoviral destination vector (pAd/PL-DEST) were established with LR Clonase II (Invitrogen). The control adenovirus (Ad-LacZ, S36A) was obtained from R.H. Unger (University of Texas Southwestern Medical Center, Dallas, TX, USA). The virus (100 MOI) was added to the cells and incubated for $4 \mathrm{~h}$ at $37^{\circ} \mathrm{C}$. After conventional culturing of the cells for $48 \mathrm{~h}$, the cells were collected to perform the experiment.

MTT assay for cell proliferation. The viability of cultured cells was determined by assaying the reduction of 3-(4,5-dimethylthiazol-2-yl)-2,5-diphenyltetrazolium bromide (MTT) to formazan. After transfection with the PIN adenovirus (Ad-PIN1) or control adenovirus (Ad-LacZ), the cells were washed twice in 96-well plates with phosphate-buffered saline (PBS), and MTT $(100 \mu \mathrm{g} / 100 \mu \mathrm{l}$ of PBS) was added to each well. The cells were then incubated at $37^{\circ} \mathrm{C}$ for $2 \mathrm{~h}$ and DMSO $(100 \mu \mathrm{l})$ was added to dissolve the formazan crystals. Absorbance was measured at $570 \mathrm{~nm}$ with a Spectra Max Plus model (Molecular Devices, Sunnyvale, CA, USA). In the juglone treatment group, cells were incubated with different doses of juglone $(1,10,20$ or $50 \mu \mathrm{M}$ ) for 6,12 or $24 \mathrm{~h}$ after virus treatment. Other processes were the same as the ones described above.

Cell cycle analysis. Cells $\left(1-5 \times 10^{5}\right)$ in 6-well plates were incubated with Ad-PIN1 or AD-LacZ for $4 \mathrm{~h}$. Cells were treated with or without juglone for $48 \mathrm{~h}$. After trypsinization and collection, cells were washed 3 times with PBS and fixed in $70 \%$ ethanol overnight at $4^{\circ} \mathrm{C}$. Cells were then washed 3 times in PBS with $0.1 \%$ BSA. Cells were incubated with $5 \mathrm{mg} / \mathrm{ml}$ of RNase A (DNase free) and $50 \mathrm{mg} / \mathrm{ml}$ of PI for $90 \mathrm{~min}$ at $4^{\circ} \mathrm{C}$. The percentages of cells in different phases of the cell cycle were measured with a FACStar flow cytometer (BectonDickinson, San Jose, CA, USA).

Histology and immunohistochemistry. Tissue sections (5 $\mu \mathrm{m})$ were stained with hematoxylin and eosin (H\&E) for analysis under a light microscopy. Immunohistochemical staining was performed with the Dako Envision system (Dako, Carpinteria, CA, USA), which uses dextran polymers conjugated with horseradish peroxidase to avoid contamination with endogenous biotin. The paraffin samples were warmed at $55^{\circ} \mathrm{C}$ for $30 \mathrm{~min}$. After deparaffinization, tissue sections were treated using a microwave antigen retrieval procedure in $0.01 \mathrm{M}$ sodium citrate buffer (pH 6.0) and immunostained with antibody against PIN1.

Western blot analysis. After collection, cells were placed in lysis buffer (M-PER; Thermo Scientific) for $30 \mathrm{~min}$ and then centrifuged at $13,200 \mathrm{xg}$ for $30 \mathrm{~min}$ at $4^{\circ} \mathrm{C}$. The supernatant was stored at $-80^{\circ} \mathrm{C}$ until use. The fresh osteosarcoma tissues $(200 \mathrm{mg})$ were cut into small pieces and put in lysis buffer (M-PER), homogenized, then centrifuged at $13,200 \mathrm{x}$ g for $15 \mathrm{~min}$ at $4^{\circ} \mathrm{C}$. The supernatant was stored at $-80^{\circ} \mathrm{C}$ until use. Protein concentration was determined using the Bradford method. The lysates $(20 \mu \mathrm{g} /$ lane) were resolved in a $10 \%$ SDS-PAGE gel depending on the target protein sizes and the resolved proteins were transferred onto Immobilon-PVDF membranes (Millipore, Billerica, MA, USA) by electroblotting. Immunoblotting was performed using a primary antibody directed specifically against PIN1 (Santa Cruz 

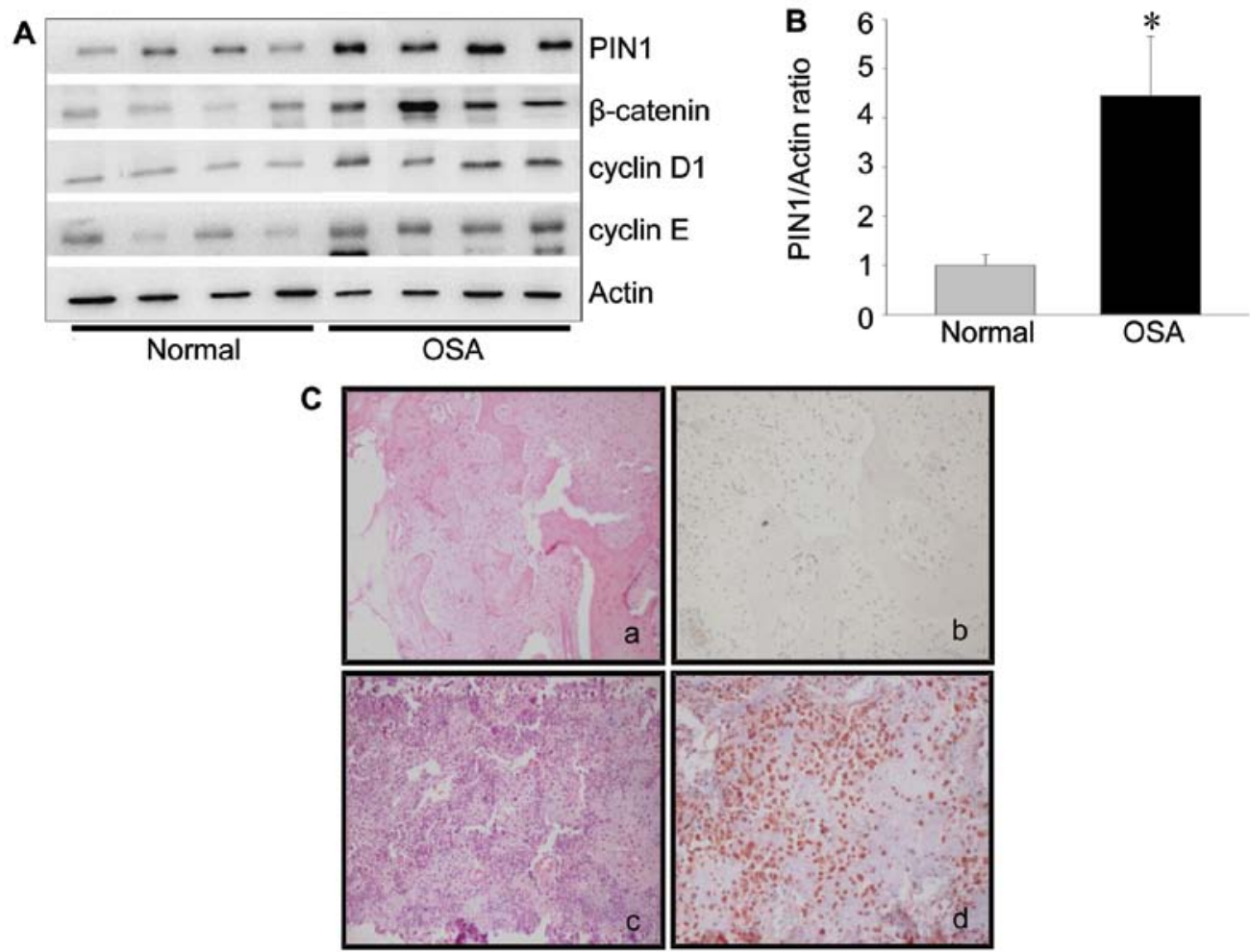

Figure 1. Expression of PIN1 in human osteosarcoma tissues. (A) Expression of PIN1, $\beta$-catenin, cyclin D1 and E proteins in normal or osteosarcoma tissues (OSA) and (B) densitometric analysis of each protein. (C) Expression of PIN1 in (a and b) normal or (c and d) osteosarcoma tissues by H\&E and immunohistochemical staining. "Significantly different from the normal tissue, $\mathrm{P}<0.05$.

Biotechnology, Santa Cruz, CA, USA) at a dilution of 1:2,000. A $\beta$-actin monoclonal antibody (Santa Cruz Biotechnology) was used as a control to demonstrate equal loading and transfer. The membranes were incubated with secondary antibodies at a dilution of 1:2,000. The membrane blots were developed using an Amersham ECL ${ }^{\mathrm{TM}}$ Advance Western Blotting Detection kit (Amersham, Arlington Heights, IL, USA).

Statistical analysis. Statistical analysis of the data was performed using ANOVA and Duncan's test. P-value $<0.05$ was considered to indicate a statistically significant difference.

\section{Results}

PIN1 is overexpressed in human osteosarcoma tissues. To assess the effect of PIN1 on osteosarcoma formation and development, PIN1 expression was assessed in human osteosarcoma and normal tissues by immunohistochemistry and western blotting. In the western blotting study, the expression levels of PIN1 protein were 4-fold higher in osteosarcoma tissues compared with normal tissues (Fig. 1A and B). The cyclin D1 level was also significantly increased in osteosarcoma tissues (Fig. 1A). Meanwhile, the expression of proteins upstream of cyclin D1, including $\beta$-catenin and cyclin $\mathrm{E}$ were also significantly increased in osteosarcoma tissues (Fig. 1A). In the immunohistochemical study, the PIN1 expression was observed in the nucleus and cytoplasm of human osteosarcoma tissues (Fig. 1C).

PIN1 induces cell proliferation in human osteosarcoma cells. After transfection of the PIN1 adenovirus for $24 \mathrm{~h}$, cellular proliferation was assessed using human osteosarcoma cells,
U2-OS and MG63. MTT analysis found that PIN1 overexpression increased the cellular proliferation by $\sim 118 \pm 5.5 \%$ in $12 \mathrm{~h}$ and $121 \pm 7.5 \%$ in $24 \mathrm{~h}$ (Fig. 2A and B). When the PIN1 inhibitor juglone was added, the effects of PIN1 overexpression were abolished (Fig. 2A and B). Juglone also induced cell death of the U2-OS and MG63 cells in a concentration- and time-dependent manner (Fig. 2C-F).

PIN1 induces the transition of the $G_{0} / G_{1}$ to the $G_{2} / M$ phase in human osteosarcoma cells. Following the overexpression of PIN1, cell cycle distribution was determined by FACS analysis (Fig. 3A). PIN1 overexpression decreased the distribution of $G_{0} / G_{1}$ phase cells to $35.26 \pm 3.41 \%$, whereas $G_{0} / G_{1}$ phase cells of the control group accounted for $50.36 \pm 3.27 \%$ of the total population. The percentages of cells at the $\mathrm{S}$ and $\mathrm{G}_{2} / \mathrm{M}$ phases were $18.52 \pm 2.52$ and $32.44 \pm 3.83 \%$, respectively, in PIN1-overexpressed cells, while the same cell populations comprised $12.17 \pm 2.31$ and $18.33 \pm 1.96 \%$, respectively, in the control cells. There was no change in the control virustransfected cells: $\mathrm{G}_{0} / \mathrm{G}_{1}(41.81 \pm 3.75 \%), \mathrm{S}(13.22 \pm 3.09 \%)$ and $\mathrm{G}_{2} / \mathrm{M}(25.35 \pm 3.18 \%)$. Juglone treatment abolished all effects of PIN1 overexpression.

PIN1 modulates the protein levels of $G_{2} / M$ phase regulators. Consistent with increased cell growth, PIN1 induced the transition from $G_{0} / G_{1}$ to $G_{2} / M$ in the U2-OS cells. We assessed the effect of PIN1 on cell cycle regulatory molecules that play important roles in the cell cycle transition. The levels of cyclins D1 and E and their associated cyclin-dependent kinases, CDK4 and CDK6, were increased in PIN1-overexpressed cells (Fig. 4). However, the expression of the cell cycle inhibitor, 
A
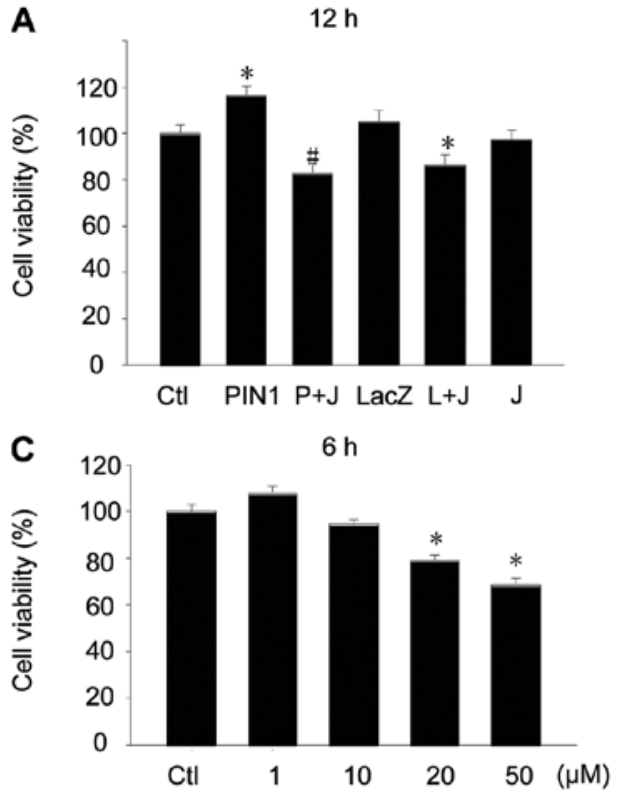

E

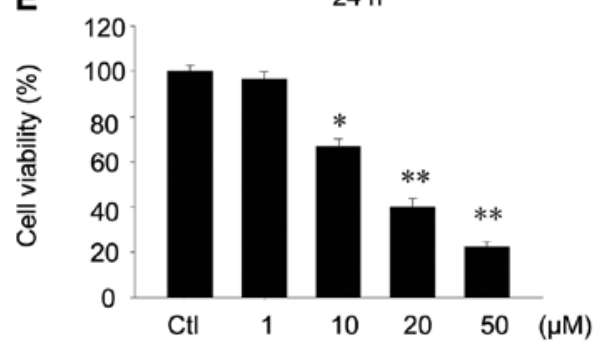

B
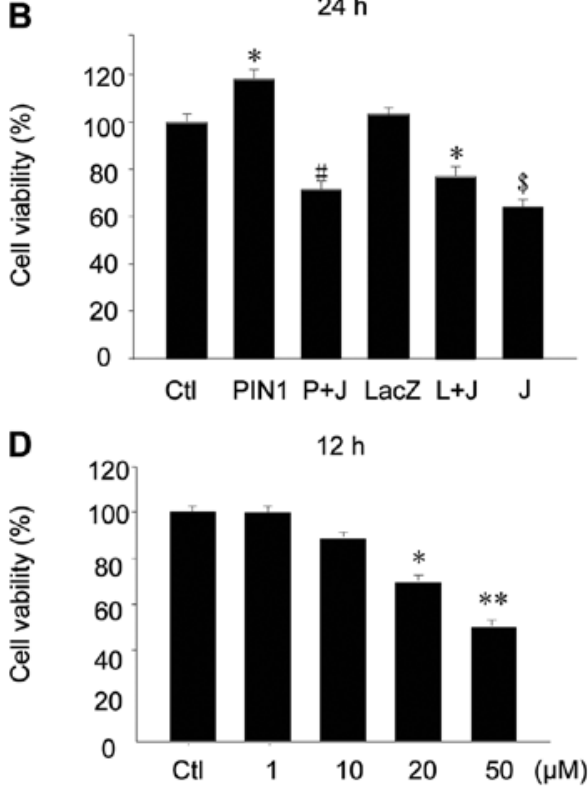

$\mathbf{F}$

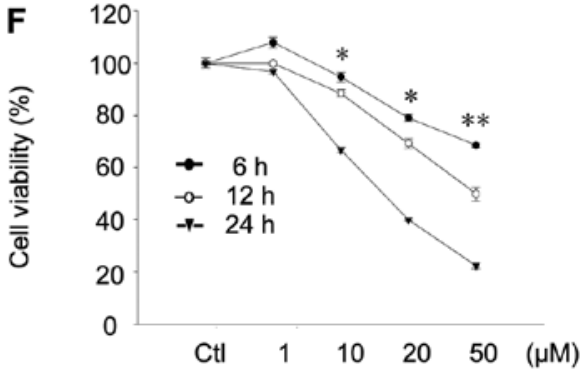

Figure 2. Effects of PIN1 and inhibitor on cell proliferation in human osteosarcoma U2-OS and MG63 cells. Cells were infected with Ad-PIN1 or Ad-LacZ for 24 h and then treated with $10 \mu \mathrm{M}$ juglone for (A) $12 \mathrm{~h}$ or (B) $24 \mathrm{~h}$. Concentration- and time-dependent effects of juglone on cell viability. Cells were treated with different concentrations of juglone $(1,10,20$ or $50 \mu \mathrm{M})$ for (C) $6 \mathrm{~h},(\mathrm{D}) 12 \mathrm{~h}$ or (E) $24 \mathrm{~h}$ and (F) time-dependent effects of juglone on cell viability are displayed. Cell viability was determined by the MTT assay and is presented as a calculated percentage of viable cells. Each point represents the mean \pm SEM of 3 determinations. ${ }^{*} \mathrm{P}<0.05$; ${ }^{* *} \mathrm{P}<0.01$ vs. Ad-LacZ-treated group; ${ }^{*} \mathrm{P}<0.05$ vs. Ad-PIN1-treated group; ${ }^{\$} \mathrm{P}<0.05$ vs. control group. Ctl, control; P + J, PIN1 + juglone; L + J, Ad-LacZ + juglone; J, juglone.

p21, was decreased in PIN1 overexpressed cells. Juglone abolished PIN1-mediated changes in cell cycle protein expression.

\section{Discussion}

PIN1, a peptidyl-prolyl cis-trans isomerase (PPIase), binds to phosphorylated Ser/Thr-Pro motifs and catalyzes a conformational change in its target, thereby altering the conformation of its substrates (2). At the molecular level, protein-protein interactions are mediated by specialized molecules consisting of dedicated signaling domains and their cognate binding partners. By interacting with these domains, PIN1 has the ability to modify protein function. For this reason, PIN1 has been implicated as a key regulator in a number of cell events, including cell cycle progression, transcription, cell proliferation and apoptosis $(10,11)$. PIN1 was originally identified as a binding partner for cell cycle proteins, several transcriptional factors and cell cycle regulatory proteins. Given its ability to regulate these proteins, PIN1 is suggested to act as a 'timer' in cell cycle progression (4).

Previous studies indicated that PIN1 is overexpressed in a number of diseases. Subsequent studies confirmed that the expression levels of PIN1 are increased in tissues of various carcinoma and cancer cell lines $(9,18-24)$. A study using
60 different types of human tumors indicated that tumor tissues have higher PIN1 protein levels compared with corresponding normal tissues (19). PIN1 overexpression has been considered an indicator of prognosis in several types of cancers, including lung (25) and colon cancers (24). In some cases, the overexpression of PIN1 indicates a short tumor recurrence period following treatment (18). In the current study, we performed immunohistochemistry to examine PIN1 expression in normal and osteosarcoma human tissues. In 8 human tissue samples, PIN1 expression was very low in the normal tissues, whereas high levels of PIN1 were observed in osteosarcoma tissues. Consistent with this finding, western blot data demonstrated that PIN1 levels were 4-fold higher in osteosarcoma tissues compared to the normal tissues.

PIN1 expression is closely correlated with cyclin D1, $\beta$-catenin and cyclin $E$ levels, which are intrinsically involved in oncogenesis $(16,21)$. The correlation between PIN1 and cyclin D1 expression suggests that PIN1 expression is not only highly regulated, but that it also plays specific roles in oncogenesis. To examine this possibility, we transiently overexpressed PIN1 in 2 osteosarcoma cell lines, MG-63 and U2-OS and then examined its effects on cell proliferation. Results demonstrated that PIN1 overexpression increased cell 

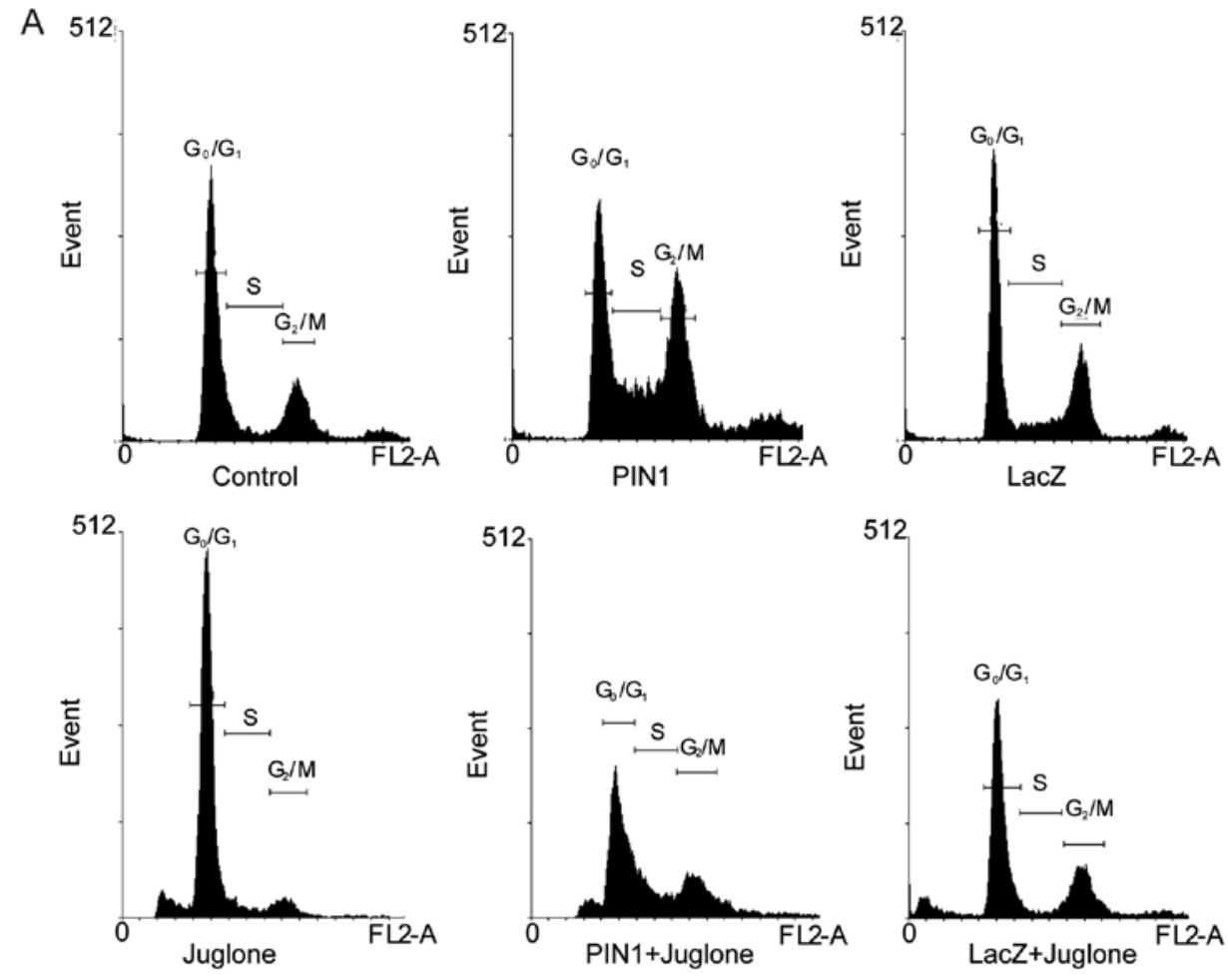

B

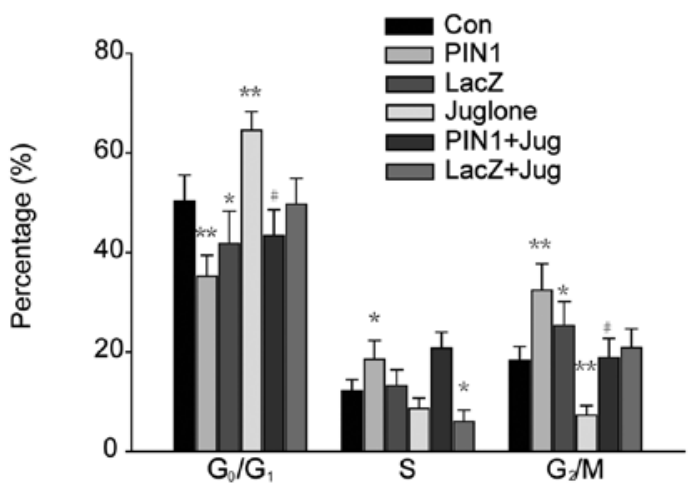

Figure 3. Effects of PIN1 on the cell cycle patterns of U2-OS cells detected by flow cytometry. U2-OS cells were infected with Ad-PIN1 or Ad-LacZ for $24 \mathrm{~h}$. Cells were collected and processed for an analysis of cell cycle regulation. (A) Representative histograms of cell cycle distribution in all groups are demonstrated. (B) The data points in the graph are the means \pm SEM of 3 independent experiments in U2-OS cells. "P<0.05, ${ }^{* *} \mathrm{P}<0.01$ vs. Ad-LacZ-treated group; ${ }^{\prime} \mathrm{P}<0.05$ vs. Ad-PIN1 treated group.

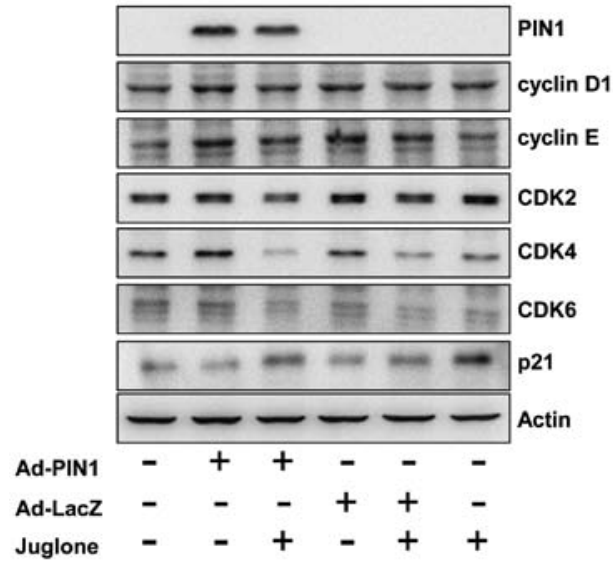

Figure 4. Effects of PIN1 on cell cycle-related proteins. U2-OS cells $\left(1 \times 10^{6}\right)$ were treated with PIN1 adenovirus or $10 \mu \mathrm{M}$ juglone for $24 \mathrm{~h}$ and cyclins D1 and E, CDKs 2, 4 and 6 and p21 were examined by western blotting. A representative result is presented for each from at least 3 separate experiments yielding similar results. proliferation and that juglone decreased cell proliferation. These results revealed that PIN1 causes cell cycle progression. This conclusion is further supported by our observation of increased cell numbers in the $\mathrm{G}_{2} / \mathrm{M}$ phase of the cell cycle.

Cell proliferation is strictly governed by a family of protein kinase complexes that are controlled, in part, by CDKs and their binding partners, the cyclins $(26,27)$. Normally, these complexes are activated by the passage of a specific checkpoint and then cells progress through the phases of the cell cycle. Any defect in this machinery causes an alteration in cell cycle regulation that may result in unwanted cellular proliferation culminating in the development of cancer $(28,29)$. Mechanistically, PIN1 increases cyclin D1 gene expression by activating $\beta$-catenin/T-cell transcription factor (TCF) (9) and directly binds to cyclin D1 to increase its stability (8). Furthermore, PIN1 regulates Ki67, cyclin E and c-MYC (30,31). Cyclins D1 and E, along with CDKs 2,4 and 6 , are the driving forces behind the $G_{1}-S$ transition in the cell cycle, whereas p21 induces the $G_{1}$ and $G_{2} / M$ phase cell 
cycle arrest (32). In our study, significant increases in the expression of cyclins D1 and E and CDKs 2, 4 and 6 were observed in the PIN1-transfected U2-OS cells. Increases in these checkpoint proteins lead to uncontrolled cell growth and aberrant cell function, with the cells quickly traversing the $G_{0}-G_{1}$ and/ or $\mathrm{G}_{1}-\mathrm{S}$ transitions. Thus, PIN1, by regulating the expression of cyclin D1, cyclin E and CDKs, has the ability to promote tumor growth. Recently, PIN1 has been suggested to promote the epithelial-mesenchymal transition (33), often regarded as the initiation of a metastatic and aggressive cancer. However, more detailed studies are required to elucidate the mechanism underlying PIN1-induced metastasis in osteosarcoma cell lines.

Osteosarcoma continues to be the leading cause of bone cancer that mainly occurs in adolescent and young adults. Delayed diagnosis and lack of effective therapeutics cause osteosarcoma to recur and spread to distant sites (17). Although surgery offers the best treatment option, this cancer is often not detected early enough for surgery to be effective. The possibility of reducing the incidence and the burden of this cancer is what makes the search for promising advances in diagnosis and treatment of the utmost importance. The present study focused on the prognostic marker that contributes to osteosarcoma carcinogenesis and the effects of PIN1 on tumorigenesis. This study demonstrates that PIN1 may be a suitable prognostic marker for osteosarcoma and that it markedly induces cell proliferation through the upregulation of the CDK/cyclin complex and the downregulation of p21 expression. Taken together, these results suggest the possible application of PIN1 as a target for cancer prevention or therapy in osteosarcoma.

\section{Acknowledgements}

This study was supported by the Basic Science Research Program through the National Research Foundation of Korea (NRF) funded by the Ministry of Education, Science and Technology (2010-0021514).

\section{References}

1. Galat A: Peptidylprolyl cis/trans isomerases (immunophilins): biological diversity - targets - functions. Curr Top Med Chem 3 : 1315-1347, 2003.

2. Lu KP, Suizu F, Zhou XZ, Finn G, Lam P and Wulf G: Targeting carcinogenesis: a role for the prolyl isomerase Pin1? Mol Carcinog 45: 397-402, 2006

3. Lu KP, Hanes SD and Hunter T: A human peptidyl-prolyl isomerase essential for regulation of mitosis. Nature 380: 544-547, 1996.

4. Blume-Jensen P and Hunter T: Oncogenic kinase signalling. Nature 411: 355-365, 2001.

5. Hunter T: Prolyl isomerases and nuclear function. Cell 92: 141-143, 1998.

6. Berger M, Stahl N, Del Sal G and Haupt Y: Mutations in proline 82 of p53 impair its activation by Pin1 and Chk2 in response to DNA damage. Mol Cell Biol 25: 5380-5388, 2005.

7. Albert A, Lavoie S and Vincent M: A hyperphosphorylated form of RNA polymerase II is the major interphase antigen of the phosphoprotein antibody MPM-2 and interacts with the peptidylprolyl isomerase Pin1. J Cell Sci 112: 2493-2500, 1999.

8. Liou YC, Ryo A, Huang HK, et al: Loss of Pin1 function in the mouse causes phenotypes resembling cyclin D1-null phenotypes. Proc Natl Acad Sci USA 99: 1335-1340, 2002.

9. Ryo A, Nakamura M, Wulf G, Liou YC and Lu KP: Pin1 regulates turnover and subcellular localization of beta-catenin by inhibiting its interaction with APC. Nat Cell Biol 3: 793-801, 2001.

10. Yaffe MB, Schutkowski M, Shen M, et al: Sequence-specific and phosphorylation-dependent proline isomerization: a potential mitotic regulatory mechanism. Science 278: 1957-1960, 1997.
11. Zhou XZ, Kops O, Werner A, et al: Pin1-dependent prolyl isomerization regulates dephosphorylation of Cdc25C and tau proteins. Mol Cell 6: 873-883, 2000

12. Fujimori F, Takahashi K, Uchida $C$ and Uchida T: Mice lacking Pin1 develop normally, but are defective in entering cell cycle from G(0) arrest. Biochem Biophys Res Commun 265: 658-663, 1999.

13. Maleszka R, Hanes SD, Hackett RL, de Couet HG and Miklos GL: The Drosophila melanogaster dodo (dod) gene, conserved in humans, is functionally interchangeable with the ESS1 cell division gene of Saccharomyces cerevisiae. Proc Natl Acad Sci USA 93: 447-451, 1996.

14. Hsu T, McRackan D, Vincent TS and Gert de Couet H: Drosophila Pin1 prolyl isomerase Dodo is a MAP kinase signal responder during oogenesis. Nat Cell Biol 3: 538-543, 2001.

15. Wulf GM, Ryo A, Wulf GG, et al: Pin1 is overexpressed in breast cancer and cooperates with Ras signaling in increasing the transcriptional activity of c-Jun towards cyclin D1. EMBO J 20: 3459-3472, 2001

16. Miyashita H, Uchida T, Mori S, Echigo S and Motegi K: Expression status of Pin 1 and cyclins in oral squamous cell carcinoma: Pin1 correlates with cyclin D1 mRNA expression and clinical significance of cyclins. Oncol Rep 10: 1045-1048, 2003.

17. Lee JH, Song MY, Song EK, et al: Overexpression of SIRT1 protects pancreatic beta-cells against cytokine toxicity by suppressing the nuclear factor-kappaB signaling pathway. Diabetes 58: 344-351, 2009.

18. Ayala G, Wang D, Wulf G, et al: The prolyl isomerase Pin1 is a novel prognostic marker in human prostate cancer. Cancer Res 63: 6244-6251, 2003.

19. Bao L, Kimzey A, Sauter G, Sowadski JM, Lu KP and Wang DG: Prevalent overexpression of prolyl isomerase Pin1 in human cancers. Am J Pathol 164: 1727-1737, 2004.

20. Chen SY, Wulf G, Zhou XZ, Rubin MA, Lu KP and Balk SP. Activation of beta-catenin signaling in prostate cancer by peptidyl-prolyl isomerase Pin1-mediated abrogation of the androgen receptor-beta-catenin interaction. Mol Cell Biol 26: 929-939, 2006.

21. Fukuchi M, Fukai Y, Kimura H, et al: Prolyl isomerase Pin1 expression predicts prognosis in patients with esophageal squamous cell carcinoma and correlates with cyclin D1 expression. Int J Oncol 29: 329-334, 2006.

22. Kim CJ, Cho YG, Park YG, et al: Pin1 overexpression in colorectal cancer and its correlation with aberrant beta-catenin expression. World J Gastroenterol 11: 5006-5009, 2005.

23. Kokkinakis DM, Liu X and Neuner RD: Modulation of cell cycle and gene expression in pancreatic tumor cell lines by methionine deprivation (methionine stress): implications to the therapy of pancreatic adenocarcinoma. Mol Cancer Ther 4: 1338-1348, 2005.

24. Kuramochi J, Arai T, Ikeda S, Kumagai J, Uetake H and Sugihara K: High Pin1 expression is associated with tumor progression in colorectal cancer. J Surg Oncol 94: 155-160, 2006.

25. He J, Zhou F, Shao K, et al: Overexpression of Pin1 in non-small cell lung cancer (NSCLC) and its correlation with lymph node metastases. Lung Cancer 56: 51-58, 2007.

26. Clurman BE and Roberts JM: Cell cycle and cancer. J Natl Cancer Inst 87: 1499-1501, 1995.

27. Sherr CJ: Cancer cell cycles. Science 274: 1672-1677, 1996.

28. Jacks T and Weinberg RA: Cell-cycle control and its watchman. Nature 381: 643-644, 1996.

29. Pestell RG, Albanese C, Reutens AT, Segall JE, Lee RJ and Arnold A: The cyclins and cyclin-dependent kinase inhibitors in hormonal regulation of proliferation and differentiation. Endocr Rev 20: 501-534, 1999.

30. Yeh ES, Lew BO and Means AR: The loss of PIN1 deregulates cyclin $\mathrm{E}$ and sensitizes mouse embryo fibroblasts to genomic instability. J Biol Chem 281: 241-251, 2006.

31. Yeh E, Cunningham M, Arnold $\mathrm{H}$, et al: A signalling pathway controlling c-Myc degradation that impacts oncogenic transformation of human cells. Nat Cell Biol 6: 308-318, 2004.

32. Xiong Y, Hannon GJ, Zhang H, Casso D, Kobayashi R and Beach D: p21 is a universal inhibitor of cyclin kinases. Nature 366: 701-704, 1993.

33. Kim MR, Choi HK, Cho KB, Kim HS and Kang KW: Involvement of Pin1 induction in epithelial-mesenchymal transition of tamoxifen-resistant breast cancer cells. Cancer Sci 100: 1834-1841, 2009. 